\title{
The Quantum State of an Ideal Propagating Laser Field
}

\author{
S.J. van Enk and Christopher A. Fuchs \\ Bell Labs, Lucent Technologies, 600-700 Mountain Ave, Murray Hill, NJ 07974, U.S.A.
}

(21 November, 2001)

We give a quantum information-theoretic description of an ideal propagating CW laser field and reinterpret typical quantum-optical experiments in light of this. In particular we show that contrary to recent claims [T. Rudolph and B. C. Sanders, Phys. Rev. Lett. 87, 077903 (2001)], a conventional laser can be used for quantum teleportation with continuous variables and for generating continuous-variable entanglement. Optical coherence is not required, but phase coherence is. We also show that coherent states play a priveleged role in the description of laser light.

A laser produces a stable, unidirectional, more or less monochromatic, possibly very intense light beam with well-defined coherence and polarization characteristics. These properties make a laser a wonderful tool for optics experiments, but they are all classical properties in the sense that they can be understood perfectly well using Maxwell's equations. When is the quantum state of a laser field important? As one might guess, quantum information protocols provide examples. For instance, a recent paper by Rudolph and Sanders [1] discusses an instructive case where - depending upon what the quantum state of a laser field is taken to be - a laser apparently may or may not be used to demonstrate quantum teleportation, and even may or may not be used to generate entangled quantum states. Their conclusion, however, is based on an application of the standard description of a laser field inside the laser cavity. We show here that this is insufficient to properly interpret various quantum information protocols involving lasers. As such, this provides an opportunity to deepen our understanding of what gives quantum information processing its power.

According to textbook laser theory - see for example [2, ch. 17] and [3, ch. 12] - the quantum state of the field inside a laser cavity in a steady state is well approximated by a mixed state diagonal in the photon-number basis. The expectation value of the electric field in such a state vanishes. On the other hand, many, if not all, standard optics experiments seem to be consistent with the assumption that the laser field is in a coherent state. The expectation value of the electric field in a coherent state is nonzero and has a well-defined phase and amplitude. It corresponds to a classical monochromatic light field, a solution of the classical Maxwell equations. Mølmer addressed the apparent contradiction between the two different descriptions of a laser field in [4]. There, he conjectured that no standard optics experiment has yet proved the existence of a nonzero expectation value of the electric field, and we agree with that. For instance, he shows that a standard measurement of the phase between two independent light beams emanating from cavities ini- tially in number states leads to measurement records indistinguishable from those expected of coherent states.

The following identity is crucial for at least partly understanding the connection between coherent-state descriptions and mixed-state descriptions:

$$
e^{-|\alpha|^{2}} \sum_{n} \frac{|\alpha|^{2 n}}{n !}|n\rangle\left\langle n\left|=\int \frac{\mathrm{d} \varphi}{2 \pi}\right| \alpha e^{i \varphi}\right\rangle\left\langle\alpha e^{i \varphi}\right| .
$$

The left-hand side is a mixed state diagonal in the photon-number basis with Poissonian photon-number statistics. The right-hand side is a mixture of coherent states with amplitude $|\alpha|$ and arbitrary phase. We use the short-hand $\rho_{|\alpha|}$ for this state. An experiment whose outcome does not depend on the absolute phase $\varphi$ cannot distinguish between a pure-state $|\alpha\rangle$ and a mixed-state $\rho_{|\alpha|}$ description. This observation, however, is still not sufficient to fully understand certain complicated optical experiments, as we will show by example.

If every standard optical experiment can be described just as well by a mixture of coherent states as by a particular coherent state, why should one bother to find out which description is correct? It turns out from a quantum-information theoretic point of view it might be very important to know if one has a pure coherent state and not a mixed state. For example, in [1], Rudolph and Sanders claim that teleportation with continuous variables is not possible with a mixed state, but requires a true coherent state. The main reason for their conclusion is that a mixture of two-mode squeezed states produced by a laser in a mixed state does not contain any entanglement. This is an important observation. In fact, this is a splendid example of why Eq. (11) does not completely capture the essence of experiments with laser light. Here we reexamine the question of the quantum state of a laser field from a quantum-information theoretic perspective. Our formulation clarifies why the coherent state plays a privileged and unique role in the description of propagating laser fields, and how a conventional laser can produce entanglement, even if it cannot actually produce a twomode squeezed state.

We consider an idealized situation where noise -in particular phase diffusion - and transient effects are neglected. This is sufficient for our purpose of showing that optical coherence (that is, nonzero off-diagonal matrix elements of the density matrix in the number-state basis) is not required for teleportation with continuous variables. In a separate paper [5] we will consider the quantum state of a realistic laser beam as well as a more detailed account of the idealized case. 
We model the laser as a one-sided cavity driven by a constant force (a voltage or an external field) far above threshold. As is well known from standard laser theory [2.3] the steady-state density matrix of the field inside the laser cavity is diagonal in the number-state basis with Poissonian photon number statistics. For convenience we first assume that the field is in a coherent state and calculate the quantum state of the field outside the laser cavity. Subsequently, using the identity (11), we adapt that result to find the quantum state of a propagating laser beam of a laser in the proper mixed state.

We employ standard input-output theory [3,6] to connect the quantum field inside a laser cavity to its output field. First, we separate the field modes into two parts. A single-mode annihilation operator $a$ describes the field with frequency $\omega_{0}$ inside the cavity; continuous-mode operators $b(\omega)$ describe modes with frequency $\omega$ outside the cavity. We define input and output operators by

$$
\begin{aligned}
a_{\text {in }}(t) & =\frac{-1}{\sqrt{2 \pi}} \int \mathrm{d} \omega e^{-i \omega\left(t-t_{0}\right)} b_{0}(\omega), \\
a_{\text {out }}(t) & =\frac{1}{\sqrt{2 \pi}} \int \mathrm{d} \omega e^{-i \omega\left(t-t_{1}\right)} b_{1}(\omega),
\end{aligned}
$$

where $t_{0} \rightarrow-\infty$ is a time in the far past and $t_{1} \rightarrow \infty$ is a time in the far future. The operators $b_{0}(\omega)$ and $b_{1}(\omega)$ are defined to be the Heisenberg operators $b(\omega)$ at times $t=t_{0}$ and $t=t_{1}$, respectively. The input and output operators satisfy the proper bosonic commutation relations for continuous-mode operators, $\left[a_{\text {in,out }}(t), a_{\mathrm{in}, \text { out }}^{\dagger}\left(t^{\prime}\right)\right]=$ $\delta\left(t-t^{\prime}\right)$. The relation

$$
a_{\text {in }}(t)+a_{\text {out }}(t)=\sqrt{\kappa} a(t),
$$

with $\kappa$ the decay rate of the cavity, may be regarded as a boundary condition on the electric field. When the input field is the vacuum and the field inside the cavity is a coherent state $\left|\alpha e^{-i \omega_{0} t} e^{i \phi}\right\rangle$, then according to (3) the output field is an eigenstate of $a_{\text {out }}$ with eigenvalue $\beta \equiv \sqrt{\kappa} \alpha e^{-i \omega_{0} t} e^{i \phi}$. Such a state is a continuous-mode coherent state [7] and can be written in the Schrödinger picture as

$$
|\{\beta(t)\}\rangle \equiv \exp \left(\int \mathrm{d} \omega\left[\beta(\omega) b^{\dagger}(\omega)-\beta^{*}(\omega) b(\omega)\right]\right)|\mathrm{vac}\rangle,
$$

with $|\mathrm{vac}\rangle$ the vacuum state and $\beta(\omega)$ the Fourier transform of $\beta(t)$. A continuous-mode coherent state can be described alternatively as an infinite tensor product of discrete-mode coherent states [7]. Let $\left\{\Phi_{i}(t)\right\}$ be a set of functions satisfying the orthogonality and completeness relations,

$$
\begin{aligned}
\int \mathrm{d} \tau \Phi_{i}(\tau) \Phi_{j}^{*}(\tau) & =\delta_{i j}, \\
\sum_{i} \Phi_{i}(t) \Phi_{i}^{*}\left(t^{\prime}\right) & =\delta\left(t-t^{\prime}\right) .
\end{aligned}
$$

We may then define annihilation operators $c_{i}$ (satisfying the correct bosonic commutation relations for discrete operators) according to $c_{i}=\int \mathrm{d} t \Phi_{i}^{*}(t) a_{\text {out }}(t)$. An eigenstate of $a_{\text {out }}(t)$ with eigenvalue $\beta(t)$ is also an eigenstate of $c_{i}$ with eigenvalue $\alpha_{i}=\int \mathrm{d} t \Phi_{i}^{*}(t) \beta(t)$. We now apply this formalism to describe laser light as a sequence of packets of light, each with the same duration $T$. Let the functions $\left\{\Psi_{n}(t)\right\}$ be defined by

$$
\begin{aligned}
\Psi_{n}(t) & =\frac{\exp \left(-i \omega_{0} t\right)}{\sqrt{T}} \text { for }\left|t-\frac{z_{0}}{c}-n T\right|<\frac{T}{2}, \\
& =0 \text { otherwise. }
\end{aligned}
$$

The label $z_{0}$ refers to an arbitrarily chosen reference position relative to which we partitioned the light beam into equal pieces of length $c T$. This set of functions is orthogonal and can be extended to form a complete set satisfying (5). For a CW laser described by $\beta(t)=\sqrt{\kappa} \alpha e^{-i \omega_{0} t} e^{i \phi}$ we see that each part $n$ of the light beam is in the same coherent state with eigenvalue $\alpha_{n}=\sqrt{\kappa T} \alpha e^{i \phi} \equiv \alpha_{0}$, corresponding to the modes described by (6), and $\alpha_{i}=0$ for all other modes.

Now assuming that the field inside the laser cavity is in fact a mixture $\rho_{|\alpha|}$, the quantum state of a sequence of $N$ parts corresponding to the set $\left\{\Psi_{n}\right\}$ is thus

$$
\tilde{\rho}_{N}=\int \frac{\mathrm{d} \varphi}{2 \pi}\left(\left|\alpha_{0} e^{i \varphi}\right\rangle\left\langle\alpha_{0} e^{i \varphi}\right|\right)^{\otimes N},
$$

where the integrand signifies an $N$-fold tensor product over the separate packets.

This result [8] displays a privileged role for coherent states in describing a propagating laser field: Although the quantum state inside the laser is a mixed state diagonal in the number-state basis, the quantum state of the output is not equal to a product of mixed states $\left(\rho_{\left|\alpha_{0}\right|}\right)^{\otimes N}$ (it would be for a pulsed laser). Rather it can be thought of as a mixture of $N$ copies of a coherent state, each copy with the same "unknown" phase. The real question is, is this the only such description? We would certainly not want to commit the preferred ensemble fallacy (PEF) that Rudolph and Sanders [1] rightly warn of.

The answer is given by the quantum de Finetti theorem 19,10]: Consider a source producing a sequence of systems with the property that interchanging any two of the systems will not change the joint probability distribution for the outcomes of measurements on the individuals [11]. Moreover, suppose this exchangeability property holds even when the ensemble is extended by any number of systems. The quantum de Finetti representation theorem specifies that the quantum state of any $N$ systems from such a source is necessarily of the form

$$
\tilde{\rho}_{N}=\int \mathrm{d} \rho P(\rho) \rho^{\otimes N},
$$

where $P(\rho)$ is a probability distribution over the density operators and $\mathrm{d} \rho$ is a measure on that space. Most importantly, this representation is unique up the behavior of $P(\rho)$ on a set of measure zero. 
Now contemplate performing a set of measurements on the individual systems emanating from our source. The probability distribution $P(\rho)$ in (8) must be updated according to standard Bayesian rules after the acquisition of that information 12]. Indeed, if the measurements are performed on a sufficiently large subset, and the measurements form a complete set in the space of operators, then the probability distribution will tend to a delta function $P(\rho) \rightarrow \delta\left(\rho-\rho_{0}\right)$. Comparing the state of a propagating laser field (7) with the general form (8) we see that a complete set of measurements on part of the light emanating from the laser will reduce the quantum state of the rest of the light to a pure state, and this pure state will necessarily be a coherent state. This shows the unique role of coherent states in the description of laser light.

It is true that standard optics experiments have not yet featured such complete measurements. For instance, a complete set for the case at hand would be a measurement of amplitude and absolute phase. However, recent developments [13] may make it possible to compare the phase of an optical light beam directly to the phase of a microwave field. Using this technique the only further measurement required for a complete measurement is a measurement of the absolute phase of the microwave field, which is possible electronically. This measurement would create an optical coherent state from a standard laser source for the first time. But as we will show in the next section, such a measurement does not need to be performed for most applications.

Let us now describe a typical optical experiment using (7) for a proper description of the quantum state of a laser. Mølmer in [4 showed that the detection of a phase difference between two (independent) light beams need not imply that there is a well-defined phase difference before the measurement. In particular, he showed that for light emanating from two cavities whose fields are initially in number states (whose phase is completely random), the standard setup to measure phase will indeed find a stable phase difference (though the value of this phase will be random and different from experiment to experiment). Within one experiment, it takes just a few (about three) photon detections [4] to settle on a particular value of the phase difference, after which the counting rates of the detectors remain consistent with that initial phase difference. In other words, the standard phase measurement acts almost like a perfect von Neumann measurement; the measurement will produce an eigenvalue of the corresponding observable and the state after the measurement can be described by an eigenstate of the measured variable. Generalizing this observation to continuously pumped CW lasers leads to the following simple description. Initially we have two independent laser beams $A$ and $B$ whose joint quantum state is described by

$$
\tilde{\rho}_{2 N}=\int \frac{\mathrm{d} \varphi_{A}}{2 \pi}\left(\left|\alpha_{A} e^{i \varphi_{A}}\right\rangle\left\langle\alpha_{A} e^{i \varphi_{A}}\right|\right)^{\otimes N}
$$

$$
\otimes \int \frac{\mathrm{d} \varphi_{B}}{2 \pi}\left(\left|\alpha_{B} e^{i \varphi_{B}}\right\rangle\left\langle\alpha_{B} e^{i \varphi_{B}}\right|\right)^{\otimes N}
$$

if we divide each laser beam into $N$ packages of constant duration. If the first package of each beam is used to measure a phase difference then the state of the rest of the light beams will be reduced to

$$
\begin{aligned}
\tilde{\rho}_{2 N-2}= & \int \frac{\mathrm{d} \varphi_{A}}{2 \pi}\left(\left|\alpha_{A} e^{i \varphi_{A}}\right\rangle\left\langle\alpha_{A} e^{i \varphi_{A}}\right|\right)^{\otimes(N-1)} \\
& \otimes\left(\left|\alpha_{B} e^{i\left(\phi_{0}+\varphi_{A}\right)}\right\rangle\left\langle\alpha_{B} e^{i\left(\phi_{0}+\varphi_{A}\right)}\right|\right)^{\otimes(N-1)},
\end{aligned}
$$

where we assumed the outcome of the phase measurement was $\phi_{0}$ and approximated the measurement to be sharp. The state (10) has the property that a subsequent measurement of the phase difference will reproduce the value $\phi_{0}$ : This is a kind of "phase-locking without phase." Note this would certainly not be the case if the quantum state of a laser were a product of identical mixed states of the form $\left(\rho_{|\alpha|}\right)^{\otimes N}$.

We now address the issue of teleportation with continuous variables using a two-mode squeezed state [14]. Such a state can be generated by splitting two squeezed states on a 50-50 beamsplitter. The resulting state of the two output ports is an entangled state. Denote a two-mode squeezed state generated from a coherent state with amplitude $\alpha e^{i \varphi}$ by $\left|T_{\alpha}^{A B}(\varphi)\right\rangle$, where the superscripts $A, B$ refer to two distinct modes located in different laboratories, say Alice's and Bob's. As shown in [1], the state

$$
\int \frac{\mathrm{d} \varphi}{2 \pi}\left|T_{\alpha}^{A B}(\varphi)\right\rangle\left\langle T_{\alpha}^{A B}(\varphi)\right|
$$

contains no entanglement between $A$ and $B$ : Instead, it simply denotes classical correlation between photon numbers for the two modes. Now, however, suppose that some of the remaining laser light is supplied to Alice (as for instance for the purpose of producing a local oscillator 14). The overall quantum state between Alice and Bob will then be of the form

$$
\int \frac{\mathrm{d} \varphi}{2 \pi}\left|T_{\alpha}^{A B}(\varphi)\right\rangle\left\langle T_{\alpha}^{A B}(\varphi)\right| \otimes\left(\left|\alpha_{A^{\prime}} e^{i \varphi}\right\rangle\left\langle\alpha_{A^{\prime}} e^{i \varphi}\right|\right)^{\otimes N},
$$

where $A^{\prime}$ indicates the further modes in Alice's possession. Far from being an unentangled state, this state has every bit as much entanglement as if the laser were actually a pure coherent source. It is just that the entanglement is in the form of distillable entanglement [15]. To see this, contemplate Alice doing a complete measurement on the extra laser light in her lab. With it, she will reduce the quantum state of modes $A, B$ to a true twomode squeezed state. Since these measurements are local (all measurements are performed on Alice's modes $A^{\prime}$ ), it follows there must be distillable entanglement between Alice's and Bob's modes. Although the claim in [1] that the state (11) can be produced locally by Alice and Bob is quite correct, the state (12) is entangled and cannot be so produced. 
This shows that teleportation of continuous variables is possible even with lasers in mixed states. The actual procedure used in 14 required, as was noted in [1], both Alice and Bob to use some of the light of the same laser that generated the two-mode squeezed state to perform homodyne detection. The fact that Bob shares laser light with Alice does not imply however, that they share any quantum channel over and above their original entanglement. In principle all the light in Alice and Bob's possession (both the shared two-mode squeezed state and the light for their local oscillators) was sent to them before any actual teleportation takes place.

Moreover, as pointed out in [16], such a shared resource is necessary for any teleportation protocol, irrespective of its physical implementation. For teleportation with continuous variables, Alice and Bob need to share a synchronized clock; sharing some of the laser light is a practical way of implementing this (though of course laser light is more than simply a clock ). In contrast to [1], we do not consider the presence of this resource, which acts as a phase reference, as invalidating teleportation. An independent party, Victor, who would like to verify Alice and Bob's teleportation skills, could use his own laser but has to "phase-lock" it with Alice's laser [17]. After all, Alice's claim is only that she can teleport a quantum state of a particular mode: Victor is free to choose the state to be teleported, but not the Hilbert space.

Finally, the teleportation procedure as a whole does not depend on the value of the absolute phase $\varphi$. Therefore, for teleportation to succeed, Alice does not even have to do an absolute phase measurement to actually distill the entanglement present in the state (12). Teleportation can be achieved without knowing the imagined "unknown" phase $\varphi$ arising in any PEF. In particular, Alice and Bob can teleport a quantum state handed to them by the independent third party Victor even if he is able to generate a pure coherent state or a pure entangled state. This is because the phases of both input and output state are compared to one and the same phase reference. Of course, in the actual experiment 14] no coherent state was produced and thus no coherent states were teleported. Instead it is the action of a general displacement operator (which acts on a coherent state as $\left.D_{\beta}|\alpha\rangle=\exp \left(i \operatorname{Im}\left(\beta \alpha^{*}\right)\right)|\alpha+\beta\rangle\right)$ that is teleported.

In conclusion, viewing the laser beam of a CW laser as a sequence of $N$ quantum systems leads to the following result: The quantum state of a laser beam is a mixture of $N$ copies of identical pure coherent states. Such a state is very different from $N$ copies of identical mixed states (be they mixtures of number states or of coherent states). One consequence is that appropriate measurements performed on part of a laser beam will reduce the quantum state of the rest of the laser beam to a pure coherent state. Such measurements seem in fact possible with present-day technology [13], and thus an optical coherent state may in fact be generated. No sophisticated measurement on the laser medium [4 need be contemplated to carry this out.
Most importantly, this description allows us to properly assess quantum communication protocols that rely on lasers. In particular we find that teleportation with continuous variables is possible with conventional lasers without actually having to reduce the quantum state of a laser to a coherent state.

We thank Terry Rudolph and Barry Sanders for sending us a copy of their paper, and Klaus Mølmer and the referees for extended comments.

[1] T. Rudolph and B.C. Sanders, Phys. Rev. Lett. 87, 077903 (2001).

[2] M. Sargent, M.O. Scully, and W.E. Lamb, Laser Physics, Addison-Wesley, Reading (1974).

[3] D.F. Walls and G.J. Milburn, Quantum Optics, SpringerVerlag, Berlin 1994.

[4] K. Mølmer, Phys. Rev. A 55, 3195 (1997); J. Mod. Optics 44, 1937 (1997).

[5] S.J. van Enk and C.A. Fuchs, quant-ph/0111157.

[6] M.J. Collett and C.W. Gardiner, Phys. Rev. A 30, 1386 (1984).

[7] K.J. Blow et al., Phys. Rev. A 42, 4102 (1990).

[8] There is another way to arrive at a similar result for spatial rather than temporal modes by imagining the laser beam split into an arbitrary number of lower-intensity beams by beamsplitters. The drawback to this way of deriving the form (7) is that the de Finetti theorem (which gives uniqueness to the result) cannot be applied as the ensemble of exchangeable states produced with beamsplitters cannot be extended. That is, while it is straightforward to produce any number $N$ of spatial modes with beamsplitters, once that is done, one can no longer extend the number of spatial modes by 1 without changing the original $N$ light beams. On the other hand, to create an additional temporal mode one simply waits.

[9] R.L. Hudson and G.R. Moody, Z. Wahrs. 33, 343 (1976).

[10] C.M. Caves, C.A. Fuchs, R. Schack, quant-ph/0104088;

[11] Such exchangebility of course would not hold for a phasediffusing laser. However, as already stated, consideration of the idealized case is sufficient for tackling the issues considered in 沮, 4 .

[12] R. Schack, T.A. Brun, and C.M. Caves, Phys. Rev. A 64, 014305 (2001).

[13] D.J. Jones et al., Science 288, 635 (2000).

[14] A. Furusawa et al., Science 282, 706 (1998).

[15] C.H. Bennett et al., Phys. Rev. A 54, 3824 (1996).

[16] S.J. van Enk, J. Mod. Optics 48, 2049 (2001).

[17] Actually, if there is a guarantee that both lasers have the same frequency, no phase-locking is even necessary: simply a fixed phase relation between the lasers is sufficient. 